\title{
Imaging of high-intensity focused ultrasound-induced lesions in soft biological tissue using thermoacoustic tomography
}

\author{
Xing Jin, Yuan $\mathrm{Xu}$, and Lihong V. Wang ${ }^{\mathrm{a})}$ \\ Optical Imaging Laboratory, Department of Biomedical Engineering, Texas A\&M University, \\ 3120 TAMU, College Station, Texas 77843-3120 \\ Yuncai R. Fang, Claudio I. Zanelli, and Samuel M. Howard \\ Onda Corporation, 592 Weddell Drive, Sunnyvale, California 94089
}

(Received 27 July 2004; revised 15 October 2004; accepted for publication 19 October 2004; published 10 December 2004)

\begin{abstract}
An imaging technology, thermoacoustic tomograpy (TAT), was applied to the visualization of high-intensity focused ultrasound (HIFU)-induced lesions. A single, spherically focused ultrasonic transducer, operating at a central frequency of approximately $4 \mathrm{MHz}$, was used to generate a HIFU field in fresh porcine muscle. Microwave pulses from a 3-GHz microwave generator were then employed to generate thermoacoustic sources in this tissue sample. The thermoacoustic signals were detected by an unfocused ultrasonic transducer that was scanned around the sample. To emphasize the boundaries between the lesion and its surrounding tissue, a local-tomography-type reconstruction method was applied to reconstruct the TAT images of the lesions. Good contrast was obtained between the lesion and the tissue surrounding it. Gross pathologic photographs of the tissue samples confirmed the TAT images. This work demonstrates that TAT may potentially be used to image HIFU-induced lesions in biological tissues. (C) 2005 American Association of Physicists in Medicine. [DOI: 10.1118/1.1829403]
\end{abstract}

Key words: thermoacoustic tomography, high-intensity focused ultrasound, HIFU, treatment monitoring, microwave contrast

\section{INTRODUCTION}

High-intensity focused ultrasound (HIFU) has been used as an effective minimally invasive treatment for tumors deep in the body. The objective of HIFU treatment is to use a highly focused ultrasound beam to destroy a predetermined volume of malignant tissue while minimizing, or avoiding, damage to the surrounding tissue. The HIFU beam introduces a rapid rise in temperature, which results in tissue coagulation, in the target region. ${ }^{1-4}$ Research using pathologic studies ${ }^{5}$ has shown irreversible tumor cell death and severe damage to tumor blood vessels in the treated region in human tissue in vivo. The size and shape of the induced lesions are related to the amount of ultrasonic energy delivered to the tissue. Monitoring the treated region of the target tumor, as well as the untreated region, is important for providing feedback on the treatment.

To improve the clinical effectiveness of HIFU treatments, much effort has been focused on developing effective imaging techniques to visualize the treatment process as well as determine the immediate thermal effects. Currently, HIFU ablation damage is best observed by using magnetic resonace imaging (MRI), ${ }^{6-8}$ but MRI makes the treatment cumbersome and expensive. Unfortunately, conventional pulse-echo ultrasonic imaging methods are not suitable for visualizing thermal damage because the ultrasonic backscattering coefficients of the HIFU-treated regions are not significantly different from those of the untreated surrounding regions. The changes in tissue during HIFU treatment that can be observed by B-mode ultrasound imaging are thought to be in- fluenced by gas bubbles and tissue vaporization which makes the detection unreliable. Studies on the acoustic properties of lesions have been carried out by many researchers. ${ }^{9,10}$ Their results show increases in the attenuation coefficient and the sound speed in the lesion region compared to those in untreated tissue regions. Imaging techniques based on changes in acoustic properties have been proposed to visualize HIFUinduced lesions, and while preliminary results have been obtained for ultrasonically homogeneous tissues such as liver, results for more heterogeneous tissues are still under investigation. ${ }^{11,12}$

Microwave-induced thermoacoustic tomography (TAT) uses nonionizing radiation. When tissue absorbs pulsed microwave energy, it emits acoustic waves as a result of thermoelastic expansion. The thermoacoustic signals are then recorded to obtain the energy deposition of the microwaves in the tissue. By combining the advantages of traditional microwave imaging and ultrasonic imaging, TAT yields good resolution and contrast simultaneously. The high resolution of TAT is brought about by the short wavelengths of the generated thermoacoustic waves (in our case, the wavelength is about $1.5 \mathrm{~mm}$ ). The image contrast of TAT is a result of the differences in microwave absorption rates in different tissues. ${ }^{13,14}$ Although microwave imaging also provides high contrast, it offers poor imaging resolution due to the long wavelengths of microwaves.

Electromagnetic-energy absorption in biological tissue can be measured by a specific absorption rate (SAR). ${ }^{15}$ SAR is defined as the absorbed power per unit mass. SAR has a unit of $\mathrm{W} / \mathrm{kg}$ and can be expressed as $\mathrm{SAR}=\sigma|E|^{2} / \rho$, where 
$|E|$ is the amplitude of the loscal electric field (V/m); $\sigma$ is the conductivity of the tissue $(\mathrm{S} / \mathrm{m})$; and $\rho$ is the density of the tissue $\left(\mathrm{kg} / \mathrm{m}^{3}\right)$. The SAR is the average microwave power density converted to heat. The conductivity of tissue is related to its water content and ionic concentration, and the imaginary part of the dielectric constant $\varepsilon_{\text {img }}=\sigma / \omega$, where $\omega$ is the angular frequency of the applied fields in radians/s, determines the absorption rate of the microwave energy.

Thermal ablation of tumorous tissue is associated with a decrease in water content and conductivity. There are two explanations for the loss of water in the lesion region. To kill malignant tissue with ultrasonic ablation, the temperature in the target region is elevated to $65^{\circ} \mathrm{C}$ or higher. At such high temperatures, tissue begins to coagulate and subsequently water evaporates. ${ }^{16}$ Due to water vaporization, the lesion region has less water content than the untreated region. In addition, reduced blood flow results in water loss. Experiments on breast tumor and liver tumor have shown that HIFU treatments interrupt the blood flow within tumor vessels, and, consequently, that the blood flow within the tumor vessels declines dramatically following HIFU treatments. ${ }^{16,17} \mathrm{Be}-$ cause the lesion region has less water content than the untreated area and the conductivity of this region is, therefore, lower than that of the untreated area, it absorbs less microwave energy than the untreated area. The effect of the heating on the intracellular ionic concentration, however, is still not clear. Some research has claimed that no significant changes in ionic concentration occur during or after heating. ${ }^{18}$ Because TAT can differentiate thermally induced lesions from untreated tissue based on differences in their electromagnetic properties, it has the potential to image HIFU-induced lesions.

The purpose of this paper is to establish the feasibility of using TAT to noninvasively detect the coagulated damage in tissue that is being treated. Two reconstruction algorithms are explored: a filtered backprojection and a local-tomographytype algorithm, where the latter was implemented to emphasize the boundaries between the different tissues. To verify that TAT can differentiate low water-content tissue from high water-content tissue, a sample was made by embedding low water-content fat in high water-content porcine muscle. This sample was then imaged. Subsequently, a HIFU-induced lesion in porcine muscle was produced for the purpose of evaluating the ability of TAT to detect ablation. Good contrast was obtained between the lesion and the tissue surrounding it. A comparison of the size of the lesion measured with TAT and the size measured by a gross pathologic photograph is also presented.

\section{METHODS}

\section{A. Tissue sample handling and lesion generation}

A side view of the experimental setup is shown in Fig. 1. The tissue sample was constructed by embedding a piece of fresh porcine muscle in fat. The whole tissue sample was immersed in mineral oil and placed on a base in the $X-Y$ plane. In the experiments, we carefully removed the air bubbles between the fat and muscle and fixed the relative

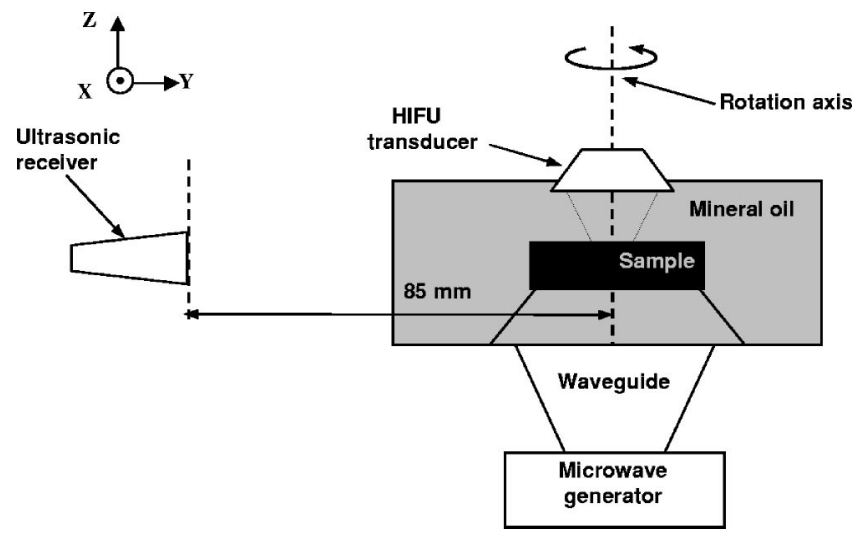

FIG. 1. Side view of the experimental setup.

position of the muscle with a thin string. The porcine muscle had a water content of $\sim 75 \%,{ }^{19}$ which is similar to the water content of cancerous tissue.

The ultrasonic receiver was an unfocused transducer (V323, Panametrics-NDT, Waltham, MA), with a central frequency of $2.25 \mathrm{MHz}$ and a diameter of $6 \mathrm{~mm}$. This receiver was scanned around the $Z$ axis, and its axis was aligned with the center of the muscle. Thermal lesions were induced in the muscle using a spherically focused transducer (Onda Corporation, Sunnyvale, CA), which irradiated the sample from above, along the $Z$ direction.

The HIFU transducer operated at a central frequency of approximately $4 \mathrm{MHz}$, with a focal length of $25 \mathrm{~mm}$. It was driven by a continuous sinusoidal voltage produced by a signal generator (DS345, Stanford Research, Sunnyvale, CA) and passed through a RF amplifier (240L, ENI). The HIFU transducer was immersed in mineral oil to provide coupling to the sample. Because the HIFU-induced lesion was formed preferentially before the focus, the distance between the HIFU transducer and the tissue sample was less than $25 \mathrm{~mm}$.

Pulsed microwave radiation was used to irradiate the tissue samples through an air-filled pyramidal horn antenna with an opening of $120 \mathrm{~mm} \times 88 \mathrm{~mm}$. The frequency of the microwaves was $3 \mathrm{GHz}$. At this frequency, the penetration depth in fat is $9 \mathrm{~cm}$, and the penetration depth in muscle is $1.2 \mathrm{~cm}$. Most other soft tissues have penetration depths between these two values. The width of the microwave pulse was $0.5 \mu \mathrm{s}$, which means that ultrasonic waves up to $\sim 2 \mathrm{MHz}$ were generated, thus providing spatial resolution on the millimeter scale. The peak power of the microwave pulse was estimated to be $20 \mathrm{~kW}$, leading to an estimated total energy per microwave pulse of about $0.01 \mathrm{~J}(20 \mathrm{~kW}$ $\times 0.5 \mu \mathrm{s}=0.01 \mathrm{~J})$. The microwave generator and the oscilloscope were synchronized by a function generator. The tissue sample was placed above the opening of the antenna as shown in Fig. 1. Several minutes after the lesion was generated in the tissue, we turned on the microwave generator and began to collect the thermoacoustic signals. The estimated microwave radiation level based on the above settings conforms to the safety requirements. ${ }^{15}$ 

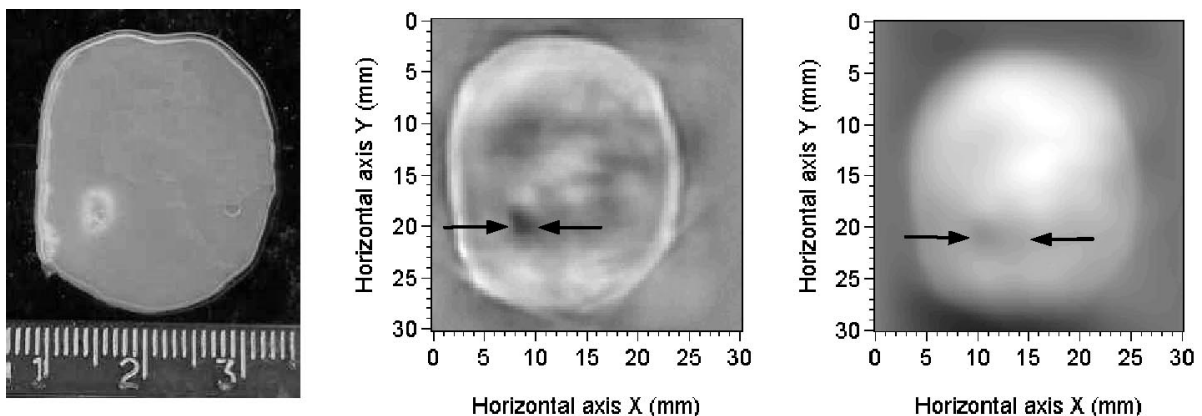

(a) (b) -1.50

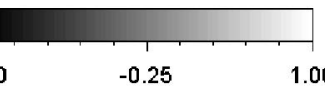

Horizontal axis $X(\mathrm{~mm})$

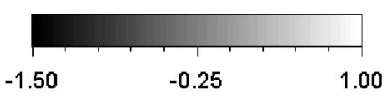

FIG. 2. (a) Gross pathologic photograph of the sample used in the experiment. (b) Reconstructed image using the local-tomography-type algorithm. (c) Reconstructed image using the approximate filtered backprojection algorithm. The lesion region is indicated by arrows.

\section{B. Reconstruction method}

The reconstruction methods are based on a previous study. ${ }^{20}$ An approximate two-dimensional filtered backprojection algorithm was applied to obtain an energy deposition image first. To emphasize the sharp details in the reconstructed image, we then used a local-tomography-type reconstruction algorithm that was also studied in the paper referenced above. In the following experiments, we reconstructed the image with full (panoramic) view data, which were collected by scanning the ultrasonic transducer around the tissue sample in a full circle. In practice, we often can obtain only limited-view data and then must use the limitedview algorithm discussed previously ${ }^{20}$ to reconstruct the image.

\section{RESULTS AND DISCUSSION}

\section{A. Experimental results}

First, the reconstructed images, using two different reconstruction methods, are compared in Fig. 2. Figure 2(a) shows a gross pathologic photograph, and Fig. 2(b) shows the results of the approximate filtered backprojection method. Because of the band-limited effect of the receiving transducer, the low-frequency components of the thermoacoustic signals detected by the receiver were filtered out. To reconstruct the image through the approximate filtered backprojection method, we estimated the ultrasound pressure by integration. The integration process resulted in only an approximation of the nonfiltered thermoacoustic signals. From Fig. 2(c), we observe that the reconstructed image is uniform due to the smoothing effect of this integration. Figure 2(b) is the result using a local-tomography-type algorithm; here the reconstructed image has better contrast. To better characterize the size and position of the lesion in the reconstructed image, we were interested in the interfaces between the muscle and the HIFU induced lesion, in which high-frequency components of the thermoacoustic signals dominated. For this reason, we used the local-tomography-type reconstruction method in our analysis. The image contrast in Fig. 2(b) is improved as a result of removing the low-frequency components and emphasizing the boundaries between the different tissues. Because of the filtering effects and our use of the local- tomography-type reconstruction method, the reconstructed image in Fig. 2(b) is not a direct energy deposition image.

Next, to examine the image contrast that results from the differences in water content in the different tissues, we began with a sample of two small pieces of fat embedded in a piece of porcine muscle. Figure 3(a) shows a photograph of the sample used. The sample consisted of two fat cylinders with a diameter of about $7 \mathrm{~mm}$ and a thickness of $6 \mathrm{~mm}$. Because the water content in fat is much lower than the water content in muscle, we expected a large contrast between the two small pieces of fat and their surrounding areas in the reconstructed image. Figure 3(b) shows the reconstructed image using the local-tomography-type reconstruction method. We observe large changes in the fat regions. This experiment verifies that image contrast is related to differences in the water content of biological tissues.

Our final step was to generate HIFU-induced lesions in the tissue and obtain TAT images of them. Because our objective was to visualize HIFU-induced lesions with TAT, we attempted to simplify the creation of the lesions by not adding a layer of normal tissue above the tissue to be treated. This should have no effect on our results in terms of microwave penetration because the microwave energy source was placed beneath the sample to force the microwave pulses to propagate through the tissue. The tissue sample was constructed of a piece of muscle surrounded by fat. Figure 4(a)
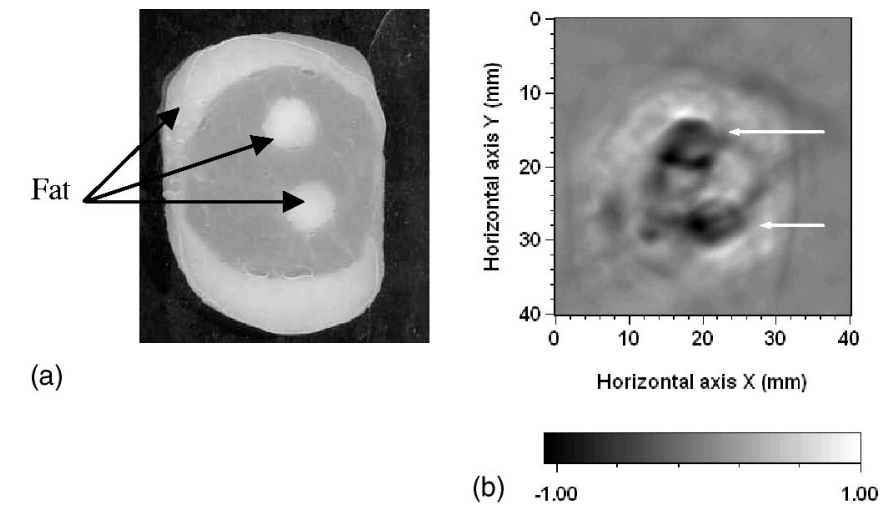

FIG. 3. (a) Photograph of the phantom used in the experiment. (b) Reconstructed image using local-tomography-type method. 

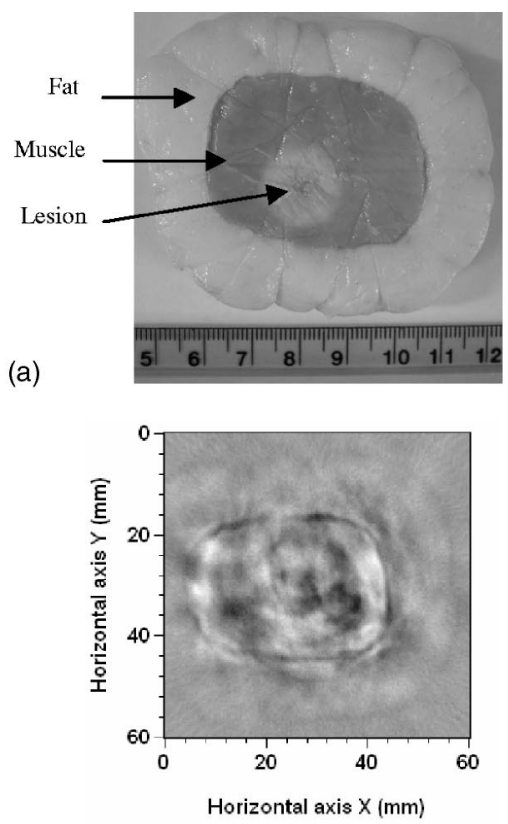

(c)
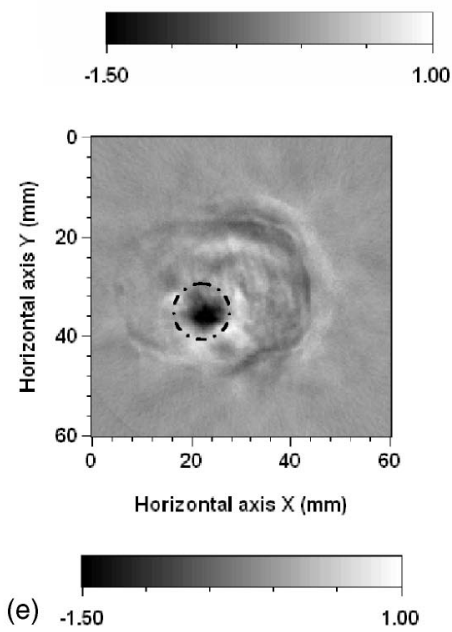

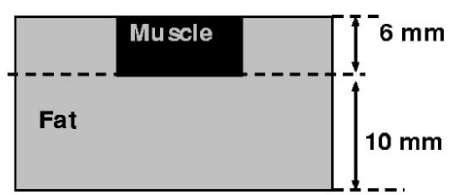

(b)

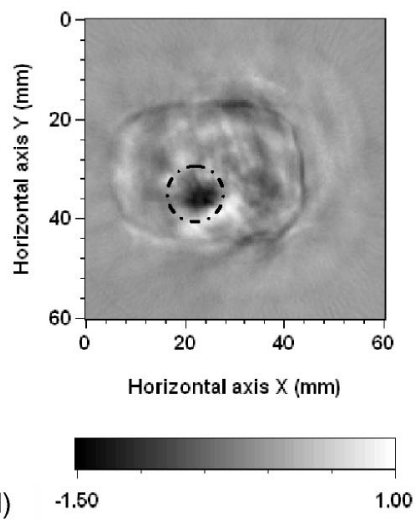

FIG. 4. (a) Gross pathologic photograph of the sample used in the experiment; the lesion was induced at a radio frequency power of $15 \mathrm{~W}$ for one and one half minutes. (b) Schematic side view of the sample. Reconstructed image using local-tomography-type reconstruction method: (c) before heating; (d) after heating. (e) The differential image obtained by subtracting the data collected before heating from the data collected after heating. The lesion regions are indicated by dashed circles. shows a photographic top view of the sample. Figure 4(b) depicts a schematic side view of the phantom used in the experiment. The approximate dimensions of the muscle were $40 \mathrm{~mm} \times 30 \mathrm{~mm}$, and the thickness of the muscle was $6 \mathrm{~mm}$. The base fat was approximately $10 \mathrm{~mm}$ in thickness. Our current microwave radiation system had limited ability to provide uniformly distributed microwave energy to the tissue sample. This limitation determined the maximum size of the sample we could use without making spatial corrections. Other factors that determined the maximum size of the sample used in the experiment included the frequency and power of the microwaves since microwave power is subject to safety requirements. The HIFU transducer heated the muscle sample from the top. The lesion was created by the HIFU transducer powered at $15 \mathrm{~W}$, corresponding to approximately $600 \mathrm{~W} / \mathrm{cm}^{2}$ at the focus for $1.5 \mathrm{~min}$. The dose of therapeutic ultrasound had been selected to induce water vaporization in the tissue. Several minutes after the formation of the lesion, we began to collect the TAT data. The thermoacoustic signals were sampled for $40 \mu$ s at a sampling rate of $50 \mathrm{MHz}$. The transducer was circularly scanned around the sample at a radius of $7.8 \mathrm{~cm}$ with a step size of $2.25^{\circ}$. At each detection angle, 200 thermoacoustic waves were averaged to abate the random noise and thus improve the signal-to-noise ratio of the detection.

The lesion showed up on the surface of the muscle as a white circle, sometimes with a central hole, which is clearly visible in Fig. 4(a). After heating, the tissue lost some of its water content due to water vaporization, which decreased accordingly its ability to absorb microwave energy. Because in this case the fat under the muscle was only about $1 \mathrm{~cm}$ in thickness, which was far less than the penetration depth in fat $(9 \mathrm{~cm}$ at $3 \mathrm{GHz})$, the fat base had no obvious influence on the quality of the reconstructed TAT image. We reconstructed the image using the local-tomography-type reconstruction algorithm. Figure $4(\mathrm{c})$ is the reconstructed image before treatment, and Fig. 4(d) is the reconstructed image after treatment. These two images do not represent energy deposition directly, because most of the low-frequency components of the thermoacoustic signals have been removed to emphasize 


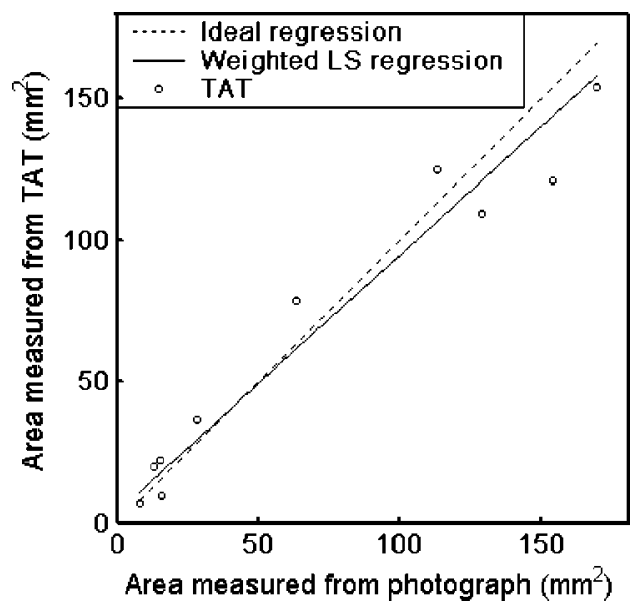

FIG. 5. Scatter plot of the area of the lesion evaluated using TAT versus the area measured from pathologic photographs. The evaluation was applied to ten lesions; the regression equation obtained using the weighted LS regression was $[\mathrm{TAT}]=0.91 \times[$ Photo $]+3.67$.

the boundaries. We estimate that the ratio of the change in microwave absorption in the lesion region to the average absorption in the surrounding normal tissue was 0.85 in this case. In other similar experiments, the ratios ranged from 0.55 to 0.95 . The lesion shown in the tissue photograph confirmed the corresponding regions measured by TAT. By subtracting the data collected before heating from the data collected after heating, but before reconstruction of the image, we obtained better image quality as shown in Fig. 4(e).

\section{B. Evaluation and characterization of the lesion}

Figure 5 compares the sizes of the lesions measured from the TAT image with the sizes measured from the photograph. The lesion in the TAT image was evaluated as the area enclosed by the half maximum intensity contour of the lesion boundary. The lesion in the photograph was defined as the heated region (colored white). The scattered circles denote the results measured from TAT. The dash line shows the ideal regression of the scattered values. The solid line represents the fit using a weighted least-squares (LS) regression. The weights are the inverse of the lesion area measured in the photograph. The resulting regression equation relating the values from TAT (denoted by TAT) and the values from the photographs (denoted by Photo) is $[$ TAT $]=0.91 \times[$ Photo] +3.67 . In other words, the size evaluated from the TAT image was smaller than the size evaluated from the pathologic photograph. As the size of the lesion becomes larger, the discrepancy becomes more obvious. It has been shown that thermal damage is associated with a significant increase in the speed of sound. ${ }^{9}$ In the reconstruction process, we assumed a uniform ultrasound speed in the tissue sample without considering a change in ultrasound speed in the lesion region. This may partly explain why the size of the lesion measured from the TAT image was smaller than the size measured from the photograph. Further work that explicitly considers the difference in ultrasound speed between lesions and the surrounding tissue can potentially improve the accuracy of this method. Another important reason for the discrepancy is the limited resolution of our system, which can be improved by decreasing the microwave pulse width and increasing the bandwidth of the receiving ultrasonic transducer.

\section{Discussion}

Two problems that exist in our current imaging system are the nonuniformity of microwave distribution and the limited bandwidth of the receiving transducer. Both have great influence on the sample size used in the experiments and the resolution of the reconstructed images.

First, we consider the effects of the nonuniformity of microwave distribution on our final results. The pyramidal horn antenna used to irradiate microwave energy into the tissue sample is fed by a $\mathrm{TE}_{10}$ waveguide. The horn antenna is tapered gradually from the waveguide dimensions to a larger aperture so as to preserve the electric field distribution of the dominant mode in the open aperture. Neglecting the effects of the currents on the exterior surfaces of the horn antenna, the electric field at the aperture of the antenna can be approximated to have the same shape as the corresponding component of the $\mathrm{TE}_{10}$ mode. The electric field of the pyramidal horn near the aperture was estimated by $E_{\xi}(\eta, \xi)$ $=E_{0,10} \sin (\pi \eta / a)$, where $E_{0,10}$ is the amplitude of the electric field of the $\mathrm{TE}_{10}$ mode, and $a$ is the dimension of the horn aperture in the $\eta$ direction. The $\eta$ direction is chosen to be along the longer dimension, and the $\xi$ direction is chosen to be along the shorter dimension. $E_{\xi}(\eta, \xi)$ depends on the $\eta$ coordinate and is independent of the $\xi$ coordinate (i.e., uniform in the $\xi$ direction). The electric field reaches its maximum at the center of the longer side, and zero at both ends. More accurate values of the transient electric field radiated by the transmitting antenna in the tissue sample can be computed by finite-difference time domain simulations. From the above analysis, we know that it is generally necessary to consider the microwave distribution in order to reconstruct an image of the tissue sample. In the current experiments, the tissue regions that we are interested in are very small compared with the antenna aperture, and we are only interested in the boundaries between different tissues. Therefore, it is safe to assume a relatively uniform distribution of microwave energy in the tissue sample. For large samples, however, this effect must be considered in order to achieve good image quality.

Next, we show the effects of the limited bandwidth of the receiving ultrasonic transducer on the received signals. The impulse response and the spectrum of the transducer are shown in Figs. 6(a) and 6(b). To illustrate the bandwidthlimited effects of the receiver, we numerically simulated the one-dimensional output piezoelectric signals of the thermoacoustic signals passing through the receiving transducer. In the simulations, we took into consideration the pulse width of the microwave pulse used in the experiments. Figure 6(c) is the temporal profile of the microwave pulse used in the experiments. Figure 6(e) shows the thermoacoustic signals 

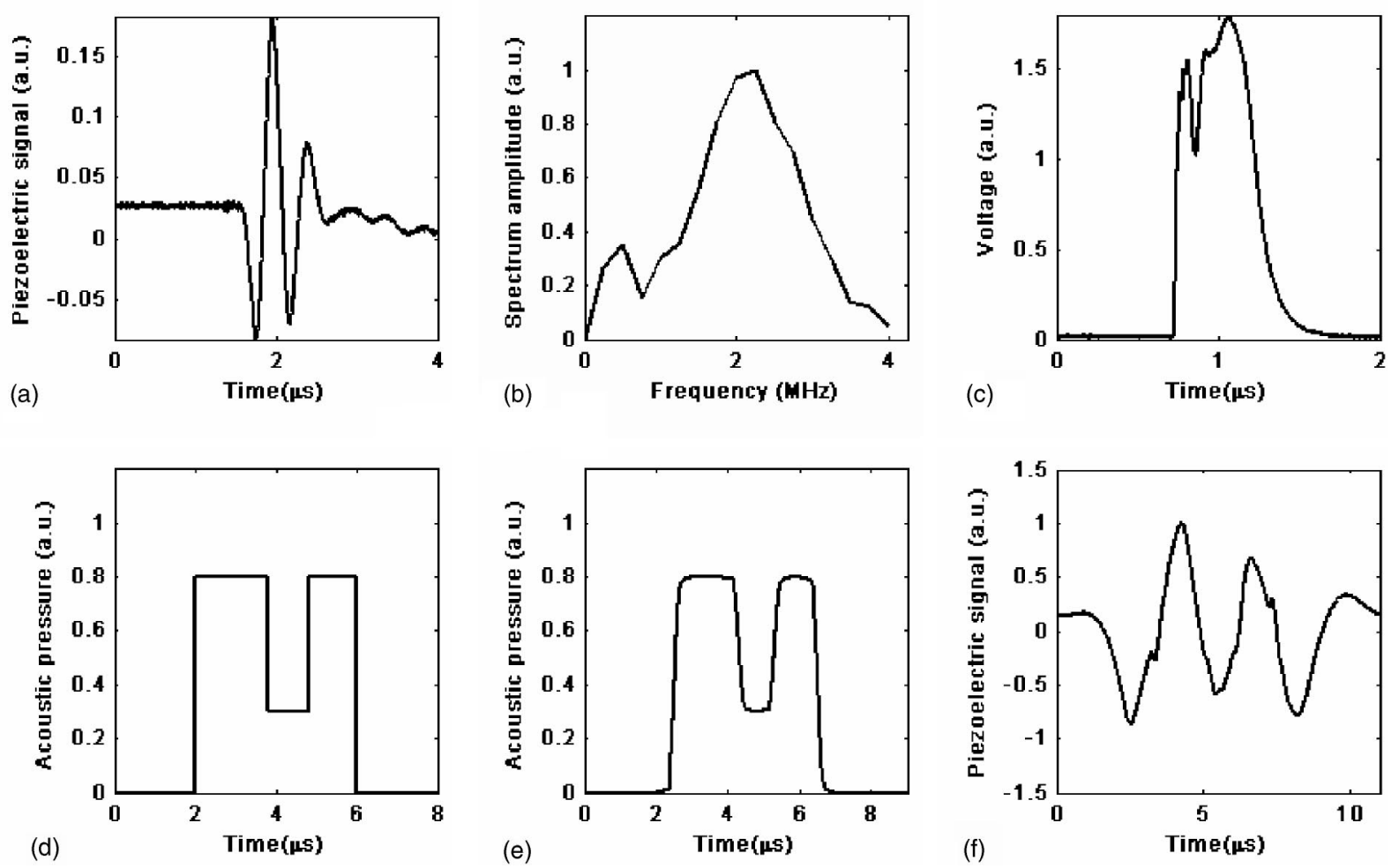

FIG. 6. Simulation of a piezoelectric signal in response to a microwave-induced thermoacoustic signal. (a) The temporal profile of the impulse response of the $2.25 \mathrm{MHz}$ transducer. (b) Spectrum of the $2.25 \mathrm{MHz}$ ultrasonic transducer. (c) Temporal profile of the microwave pulse used in the simulations. (d) Thermoacoustic signals induced by an ideal microwave pulse for a sample with a low microwave absorption region in the center. (e) Thermoacoustic signals induced by microwave pulses used in the experiments for the same samples used in (d). (f) Simulated piezoelectric signals of the $2.25 \mathrm{MHz}$ ultrasonic transducer for (e).

induced by the microwave pulse. The simulation was carried on in one dimension, rather than in two, to simplify the computations although the results can be easily extended to two dimensions. The piezoelectric output of the ultrasonic transducer in response to the thermoacoustic pressure was calculated as the convolution between the thermoacoustic pressure and the impulse response of the transducer. Because of the band-limited effects of the transducer, we expected large changes at the boundary regions in the piezoelectric output signal. The low-thermoacoustic pressure region in Fig. 6(d) was used to simulate the lesion. Figure 6(f) was the simulated piezoelectric output. We observed a deep drop in the piezoelectric signal at the lesion region. The recovery of the thermoacoustic signal through deconvolution is difficult to realize. As a reasonable simplification, in our analysis in the frequency range below $2 \mathrm{MHz}$, a differential operator was used to approximate the spectrum of the transducer.

Changes in the dielectric properties reflect HIFU-induced changes in the target region. Imaging techniques based on changes in the dielectric properties of tissue may be used to monitor the physiological changes during ultrasound treatment. Our experiments were implemented with a single ultrasonic transducer rotating in a circle to collect signals from different directions. Due to this limitation, we were unable to monitor changes in the tissue in real-time, and, consequently, changes in the dielectric properties during heating were not measured. Some research has shown that the dielectric prop- erties of tissue increase at the initial denaturation of protein, ${ }^{21-23}$ which can be explained by an increased mobility of the bound water and ions. We expect stronger microwave absorption at the initial treatment. The cavitations during the treatment, however, may complicate real-time monitoring of HIFU treatments. In the future, the use of a transducer array will significantly reduce the data collection time and provide more information on the possibility of applying TAT to the real-time monitoring of HIFU treatments.

The treatment of discrete tumors is a difficult clinical problem. When the contrast in water content between cancerous and normal tissue is large, the difference in microwave absorption is also large. Thermoacoustic tomography has been shown to be capable of detecting small tumors based on this difference in microwave absorption. ${ }^{24}$ We have demonstrated that TAT can, in addition, differentiate the tumor before and after treatment. Moreover, high-intensity focused ultrasound has been shown to be a noninvasive treatment that can induce well-defined coagulated lesions while leaving the surrounding area unaffected. The size and shape of the target region can be well controlled to focus on the individual tumor. The prospect of combining these two techniques in order to both detect tumors and apply thermal surgery during the same clinical event holds great promise for cancer treatment in the future. 


\section{CONCLUSIONS}

HIFU-induced lesions in porcine muscle were imaged with TAT. Tissue thermal damage can result in a localized change in a tissue's electromagnetic properties, and thus its ability to absorb microwave energy. Our preliminary results have shown that TAT has the capacity to visualize HIFUinduced lesions with good contrast using a localtomography-type reconstruction algorithm. The boundaries of different tissues can be imaged clearly. The size and position of lesions measured from TAT images were compared with the size and position of those measured from gross pathologic photographs. It was established that TAT can estimate the size of lesions effectively. From the preliminary studies conducted here, we conclude that TAT has the potential to provide an effective, low-cost alternative method for imaging HIFU-induced lesions.

a) Author to whom correspondence should be addressed; electronic mail LWang@tamu.edu; URL: http://oilab.tamu.edu

${ }^{1}$ C. J. Harvey, J. M. Pilcher, R. J. Eckersley, M. J. K. Blomley, and D. O. Cosgrove, “Advances in ultrasound," Clin. Radiol. 57, 157-177 (2002).

${ }^{2}$ G. T. Haar, "Acoustic surgery," Phys. Today 54(12), 29-34 (2001).

${ }^{3}$ G. T. Haar, "Ultrasound focal beam surgery," Ultrasound Med. Biol. 21(9), 1089-1100 (1995).

${ }^{4}$ G. T. Haar, D. Sinnett, and I. Rivens, "High intensity focused ultrasound-a surgical technique for the treatment of discrete liver tumors," Phys. Med. Biol. 34(11), 1743-1750 (1989).

${ }^{5}$ F. Wu, Z.-B. Wang, W.-Z. Chen, J.-Z. Zou, J. Bai, H. Zhu, K.-Q. Li, F.-L. Xie, C.-B. Jin, H.-B. Su, and G-W. Gao, "Extracorporeal focused ultrasound surgery for treatment of human solid carcinomas: early Chinese clinical experience," Ultrasound Med. Biol., 30(2), 245-260 (2004).

${ }^{6}$ K. Hynynen, O. Pomeroy, D. N. Smith, P. E. Huber, N. J. McDannold, J. Kettenbach, J. Baum, S. Singer, and F. A. Jolesz, "MR imaging-guided focused ultrasound surgery of fibroadenomas in the breast: a feasibility study," Radiology 219, 176-185 (2001).

${ }^{7}$ C. Weidensteiner, B. Quesson, B. Caire-Gana, N. Kerioui, A. Rullier, H. Trillaud, and C. T. W. Moonen, "Real-time MR temperature mapping of rabbit liver in vivo during thermal ablation," Magn. Reson. Med. 50, 322-330 (2003).

${ }^{8}$ C. M. C. Tempany, E. A. Stewart, N. McDannold, B. J. Quade, F. A. Jolesz, and K. Hynynen, "MR-imaging-guided focused ultrasound surgery of uterine leiomyomas: a feasibility study," Radiology 226, 897-905 (2003).
${ }^{9}$ N. L. Bush, I. Rivens, G. R. T. Haar, and J. C. Bamber, "Acoustic properties of lesions generated with an ultrasound therapy system," Ultrasound Med. Biol. 19(9), 789-801 (1993).

${ }^{10}$ A. E. Worthington, J. Trachtenberg, and M. D. Sherar, "Ultrasound properties of human prostate tissue during heating," Ultrasound Med. Biol. 28(10), 1311-1318 (2002).

${ }^{11}$ R. Righetti, F. Kallel, R. J. Stafford, R. E. Price, T. A. Krouskop, J. D. Hazle, and J. Ophir, "Elastographic characterization of HIFU-induced lesions in canine livers," Ultrasound Med. Biol. 25(7), 1099-1113 (1999).

${ }^{12}$ F. L. Lizzi, R. Muratore, C. X. Deng, J. A. Ketterling, S. K. Alam, S. Mikaelian, and A. Kalisz, "Radiation-force technique to monitor lesions during ultrasonic therapy," Ultrasound Med. Biol. 29(11), 1593-1605 (2003).

${ }^{13}$ G. Ku and L.-H. Wang, "Scanning thermoacoustic tomography in biological tissue," Med. Phys. 27(5), 1195-1202 (2000).

${ }^{14}$ R. A. Kruger, K. Stantz, and W. L. Kiser Jr., "Thermoacoustic CT of the breast," Proc. SPIE 4682, 521-525 (2002).

${ }^{15}$ Biological Effects of Electromagnetic Fields, P. Strauroulatis (Springer, New York, 2003).

${ }^{16}$ I. J. Rowland, I. Rivens, L. Chen, C. H. Lebozer, D. J. Collins, G. R. T. Haar, and M. O. Leach, "MRI study of hepatic tumors following high intensity focused ultrasound surgery," Br. J. Radiol., 70, 144-153 (1997).

${ }^{17}$ F. Wu, W.-Z. Chen, J. Bai, J.-Z. Zou, Z.-L. Wang, H. Zhu and Z.-B. Wang, "Tumor vessel destruction resulting from high-intensity focused ultrasoundin patients with solid malignancies," Ultrasound Med. Biol. 28(4), 535-542 (2002).

${ }^{18}$ Biological Basis of Oncologic Thermotherapy, M. Gautherie (SpringerVerlag, Berlin, 1990).

${ }^{19}$ F. A. Duck, Physical Properties of Tissue, A Comprehensive Reference Book (Academic, London, 1990).

${ }^{20} \mathrm{Y}$. Xu and L.-H. Wang, "Reconstructions in limited-view thermoacoustic tomography," Med. Phys. 31(3), 724-733 (2004).

${ }^{21}$ D. A. McRae and M. A. Esrick, "The dielectric parameters of excised EMT-6 tumors and their change during hyperthermia," Phys. Med. Biol. 37(11), 2045-2058 (1992).

${ }^{22}$ M. Pop, A. Molckovsky, L. Chin, M. C. Kolios, M. A. Jewett, and M. D. Sherar, "Change in dielectric properties at $460 \mathrm{kHz}$ of kidney and fat during heating: importance for radio-frequency thermal therapy," Phys. Med. Biol. 48, 2509-2525 (2003).

${ }^{23}$ L. Chin and M. Sherar, "Changes in dielectric properties of ex vivo bovine liver at 915 MHz during heating," Phys. Med. Biol. 46, 197-211 (2001).

${ }^{24} \mathrm{M}$. Xu and L. V. Wang, "Time-domain reconstruction for thermoacoustic tomography in a spherical geometry," IEEE Trans. Med. Imaging 21(7), 814-822 (2002). 